\title{
PROGRESS REPORT ON THE SOUTHERN PROPER MOTION PROGRAM
}

\author{
I. PLATAIS, T. M. GIRARD, V. KOZHURINA-PLATAIS \\ R. A. MENDEZ AND W. F. VAN ALTENA \\ Yale University Observatory \\ P.O. Box 208101, New Haven, CT 06520, U.S.A. \\ C. E. LOPEZ \\ Felix Aguilar Observatory \\ 5413 Chimbas, San Juan, Argentina
AND
W. Z. MA
Beijing Normal University Observatory \\ Beijing 100875, China
}

We present the status of the Yale/San Juan Southern Proper Motion program (SPM) which is the southern hemisphere extension of the Lick Observatory Northern Proper Motion program with respect to faint galaxies (Platais et al., 1993). To date, measurements and reductions in the South Galactic Pole region comprising $\approx 1000$ square-degrees on the sky have been finished. At this stage of the SPM program particular attention has been paid to the plate model choice along with an assessment of and accounting for systematic errors. For our establishing of a secondary reference frame we have noticed the presence of a potentially dangerous effect, so-called fieldindependent coma which is caused by lens decentering. We acknowledge the superb Hipparcos preliminary positions without which such analysis would be virtually impossible. The SPM data at the SGP region have also been used to constrain a multi-component Galaxy model. First results of this analysis are presented.

\section{References}

Platais, I., Girard, T.M., van Altena, W.F., López, C.E. (1993) in Workshop on Databases for Galactic Structure, eds. A.G. Davis Philip, B. Hauck and A.R. Upgren, p. 153. 